\title{
Case Report \\ Pelvic Pain and Adnexal Mass: Be Aware of Accessory and Cavitated Uterine Mass
}

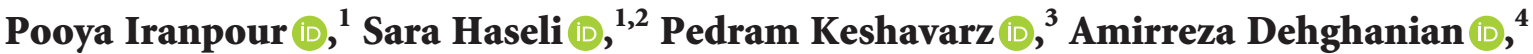 \\ and Neda Khalili $\mathbb{1 0}^{5}$ \\ ${ }^{1}$ Medical Imaging Research Center, Shiraz University of Medical Sciences, Shiraz, Iran \\ ${ }^{2}$ Chronic Respiratory Diseases Research Center, National Research Institute of Tuberculosis and Lung Diseases (NRITLD), \\ Shahid Beheshti University of Medical Sciences, Tehran, Iran \\ ${ }^{3}$ Department of Diagnostic \& Interventional Radiology of New Hospitals LTD, Tbilisi, Georgia \\ ${ }^{4}$ Department of Pathology, Shiraz University of Medical Sciences, Shiraz, Iran \\ ${ }^{5}$ School of Medicine, Tehran University of Medical Sciences, Tehran, Iran \\ Correspondence should be addressed to Sara Haseli; sarahaseli@gmail.com
}

Received 24 November 2020; Revised 19 January 2021; Accepted 30 January 2021; Published 11 February 2021

Academic Editor: Michael S. Firstenberg

Copyright (c) 2021 Pooya Iranpour et al. This is an open access article distributed under the Creative Commons Attribution License, which permits unrestricted use, distribution, and reproduction in any medium, provided the original work is properly cited.

Accessory and cavitated uterine mass (ACUM) is a rare form of Mullerian anomaly that usually presents in young females with chronic cyclic pelvic pain and/or dysmenorrhea. This clinical entity is often underdiagnosed as it may be mistaken for other differential diagnoses, such as pedunculated myoma or adnexal lesions. Imaging modalities, including ultrasonography and magnetic resonance imaging (MRI), accompanied with relevant and suspicious clinical findings are important tools in making acorrect diagnosis. To date, surgical excision of the mass remains the mainstay of treatment,which provides significant symptom relief. In this study, we present a female adolescent with chronic pelvic pain since menarche who underwent laparotomy with the presumed diagnosis of a left-sided ovarian mass. Retrospective evaluation of pelvic MR images demonstrated that the lesion was in fact an ACUM, which was further confirmed by histopathological examination.

\section{Introduction}

Accessory and cavitated uterine mass (ACUM) is a rare newly described Mullerian anomaly, which generally presents with chronic recurrent pelvic pain and/or severe dysmenorrhea in women younger than 30 years of age [1]. It is an accessory mass at the insertion of the round ligament that is lined by normal endometrium and has a dark-browncolored fluid content $[2,3]$. ACUMs are difficult to diagnose because of their broad differential diagnosis, which includes rudimentary and cavitated uterine horns such as those found in other uterine malformations (e.g., bicornuate uterus), adenomyosis with cystic or degenerated areas, degenerated leiomyomas, and essential and primary dysmenorrhea [4]. To the best of our knowledge, fewer than 60 cases of ACUM are reported in the literature at the time of writing this paper. In this case report, we describe a 14-year-old girl presenting with severe pelvic pain since her menarche. Pelvic magnetic resonance imaging (MRI) showed an apparent adnexal mass, but final histopathological examination was in favor of ACUM.

\section{Case Presentation}

A 14-year-old girl presented with chronic recurrent pelvic pain since her menarche. Her pain aggravated monthly during her menstruation; however, she had a regular and normal menstrual flow. Her personal history and family history were not significant, and she had no previous sexual history. Different kinds of anti-inflammatory medications had been prescribed for her during the past two years. The patient had also received oral contraceptive pills (OCPs) for two months, but none of the medications had been significantly effective. On physical examination, she was a 
well-developed girl with normal vital signs. She showed normal pubertal development with a height of 160 centimeters (percentile: $47 \%$ ) and weight of 48 kilograms (percentile: 43\%), and normal adrenarche and thelarche (stage 4). Pelvic examination of the external genitalia was normal with no evidence of imperforated hymen. No definite palpable mass was detected during abdominal examination. Ultrasonography was requested, which showed normal size and myometrial echogenicity of the uterus with normal endometrial thickness. There was evidence of a left-sided heterogeneous and mostly hyperechoic $35 \times 30 \mathrm{~mm}$ adnexal mass with close contact to the left ovary (Figure 1). Color Doppler showed some internal vascularity. A small right-sided simple ovarian cyst was also seen on ultrasound. The cervix and vaginal canal were unremarkable on imaging. For further characterization of the mentioned lesion, pelvic MRI was performed, which revealed a well-defined heterogeneous mass with high signal intensity on T2-weighted images and low-tointermediate signal intensity on T1-weighted images with significant enhancement after administration of gadolinium. The mass was located on the left side of the pelvic cavity, close to the left ovary and uterus; however, no connection to the uterus or ovary was detected. There was no pelvic lymphadenopathy or ascites. Based on the imaging findings, a paraovarian mass or a myoma was suspected. Subsequently, the patient underwent laparotomy, and a well-defined fleshy mass was seen on the left side of the pelvic cavity. The mass was located just under the round ligament, close to but separated from the left ovary with no adhesion to the surrounding structures. Both ovaries and uterus were normal except for a simple right-sided ovarian cyst with no endometrial implants. The uterus had two normally developed fallopian tubes with no fundal impression or contour abnormality and two normal cornua. On close observation, the uterus was completely separated from the mentioned mass. Several collateral vessels arising from the left uterine artery, which provided arterial supply to the mass, were ligated during surgery to obtain hemostasis. Frozen section revealed normal myometrial muscle with reactive endometrium. Finally, the mass was successfully resected by enucleation and incision through the serosa. While performing complete excision, a chocolate-colored fluid flowed out from the mass. The patient had an uneventful postoperative recovery and was discharged one day after surgery. She continued to be asymptomatic with complete resolution of the cyclic pelvic pain after one year of follow-up. Gross evaluation of the pelvic mass revealed a cavitated mass with a thick muscular wall, and pathological examination showed normal irregular myometrium lined by functional endometrium (Figure 2). Thus, a final diagnosis of accessory and cavitated uterine mass was made. Retrospective re-evaluation of pelvic MRI demonstrated a well-defined mass with signal intensity similar to the uterus on both T1-weighted and T2-weighted sequences with central hypersignal endometrium and normal endometrialmyometrial interface (Figures 3 and 4).

\section{Discussion}

Genitourinary development results from a complex interaction between the Wolffian ducts and the Mullerian ducts. Mullerian ducts are formed through invagination of the dorsal coelomic epithelium at about gestational week 6 . At around 10 to 12 weeks of gestation, the two Mullerian ducts, fused with each other in a craniocaudal direction, finally fuse with the urogenital sinus, forming the uterus, bilateral fallopian tubes and the upper one-third of the vagina consequently $[5,6]$.

Mullerian anomalies are deviations from normal anatomy resulting from distortion of the complex embryogenesis. Although these anomalies are usually benign conditions, they may lead to significant reproductive problems based on the degree as well as the type of maldevelopment [6]. These commonly occurring anomalies can be found in about $4-7 \%$ of the population; so, an effective and reliable classification of Mullerian anomalies can contribute to proper diagnosis and management of affected individuals [7].

Mullerian anomalies were classified by Acien and colleagues in 1992 for the first time [8]; however, the European Society of Human Reproduction and Embryology and the European Society for Gynecological Endoscopy (ESHRE/ ESGE) recently updated the classification system of female genital anomalies [7]. In this categorization, the main classes are designed on the basis of deviations from normal uterine anatomy deriving from the same embryological origin, while the clinical significance and degree of deformity are the basis for subclasses classification [7].

Uterine-like mass (ULM) is a distinct clinical entity defined as a cavitated mass lined with functional endometrium that is composed of sex hormone-sensitive smooth muscle cells, which are arranged irregularly at the periphery of the mass. These masses can be located within the uterus or anywhere else outside the uterus. ACUM, a subtype of non communicating ULM, is a rare newly described anomaly that, unlike other Mullerian anomalies, is associated with an otherwise normal uterus; hence, it should be classified as a separate entity [9]. Persistence or duplication of Mullerian duct due to a gubernaculum dysfunction at the site of round ligament attachment has been postulated as the pathogenesis of ACUM [9].

The same appearing masses have been described in the literature by different names, including cavitated adenomyoma, accessory cavitated mass, and juvenile cystic adenomyoma. However, ACUM is different from cystic adenomyosis, which usually occurs in middle-aged women, and is characterized by diffusely distributed adenomyotic foci within the uterus. Also, cystic adenomyosis lacks the normal endometrium and myometrium organization $[10,11]$.

The current criteria used for the diagnosis of ACUM includes: (1) an isolated accessory cavitated mass; (2) normal 


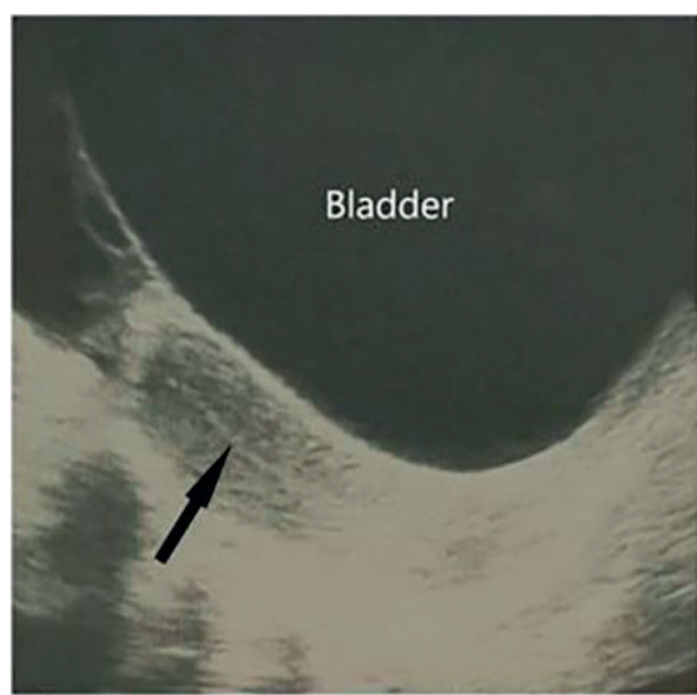

(a)

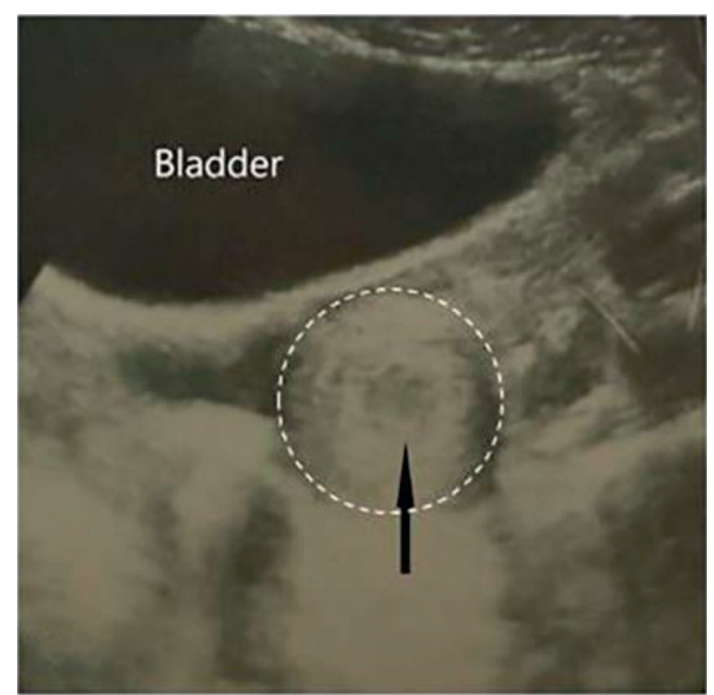

(b)

Figure 1: (a) On ultrasound, sagittal view of the pelvis shows the uterus with a normal uterine contour and normal echogenic endometrial line (arrow). (b) Axial view of the pelvis demonstrates a cross section of a mass-like lesion on the left side of the pelvic cavity, which was finally proved to be an accessory and cavitated uterine mass (arrow marks the endometrium).

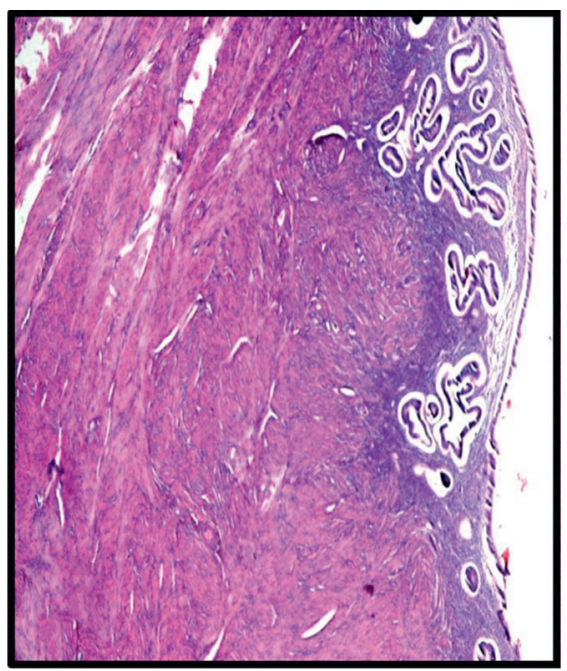

(a)

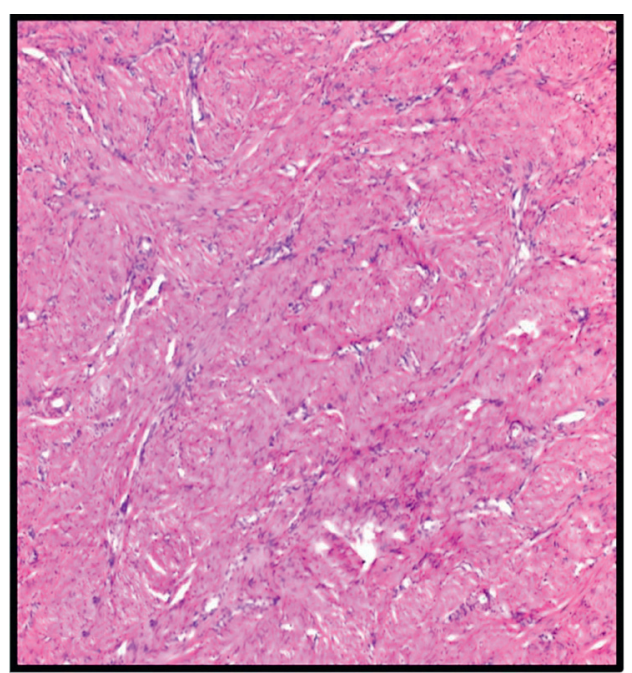

(b)

Figure 2: Histopathological examination of the pelvic mass shows (a) normal endometrial tissue in the innermost layer (hematoxylin and eosin, $\times 200$ ) surrounded by (b) thick myometrial-type muscular layer (hematoxylin and eosin, $\times 200$ ). Histopathological findings resembled a normal uterus, confirming the diagnosis of accessory and cavitated uterine mass.

uterus (endometrial cavity), tubes, and ovaries; (3) surgical case with excised mass and with pathological examination; (4) accessory cavity lined by endometrial epithelium with glands and stroma; (5) chocolate-brown-colored fluid content; and (6) no adenomyosis (if uterus removed), but there could be small foci of adenomyosis in the myometrium adjacent to the accessory cavity [9]. The present case fulfilled all of the diagnostic criteria for ACUM; in addition, since the uterus, fallopian tubes, and ovaries were normal in this patient, other Mullerian anomalies were ruled out. ACUM has multiple challenging differential diagnoses, such as cavitated uterine horn with degenerated area or rudimentary uterine horn; however, imaging modalities such as hysterosalpingography, ultrasound, and especially MRI can aid in making a proper diagnosis $[1,12,13]$. Although most of the reported ACUM cases are located on the right lateral wall of the uterus near the round ligament, about one-third of cases are adjacent to the left round ligament, as in our case [14].

Table 1 presents a summary of the main findings of patients with ACUM that have been reported in previous studies. The most common symptoms in patients with ACUM are severe dysmenorrhea and recurrent pelvic pain 


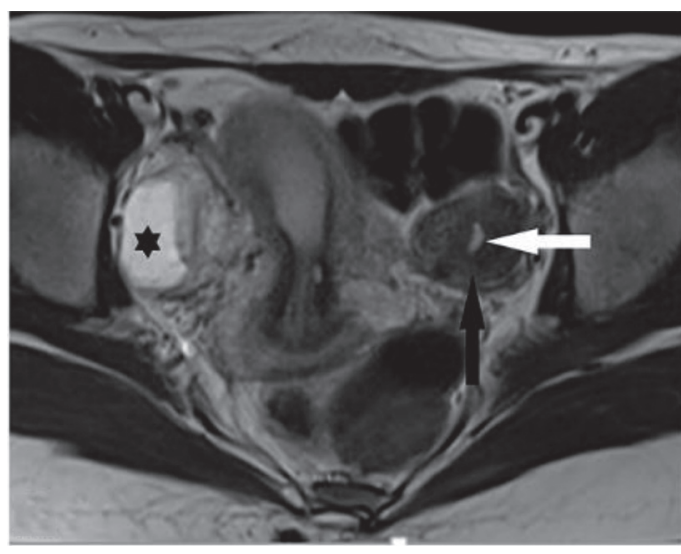

(a)

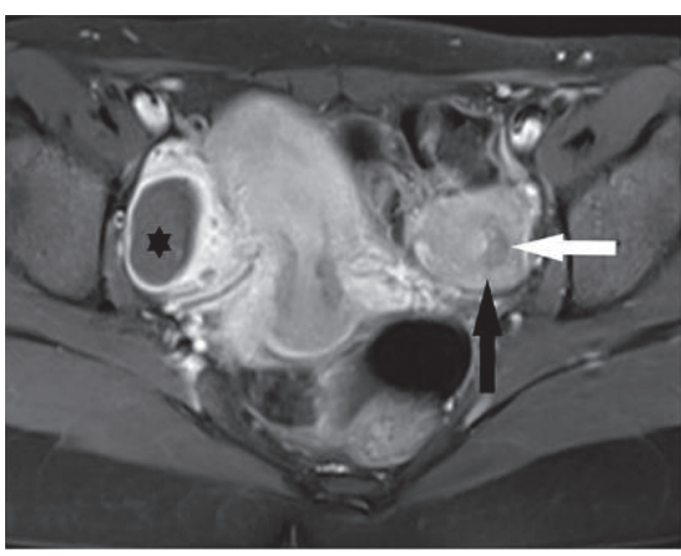

(b)

Figure 3: (a) Axial T2-weighted image of the pelvis shows a well-defined left-sided adnexal mass with signal intensity similar to the uterus (black arrow) with a central hypersignal endometrium (white arrow). (b) Axial contrast-enhanced fat-saturated T1-weighted image of the pelvis also demonstrates an enhancement similar to the uterus (black arrow). A right-sided simple ovarian cyst is also observed (asterisk). No pelvic lymphadenopathy is evident on magnetic resonance imaging.

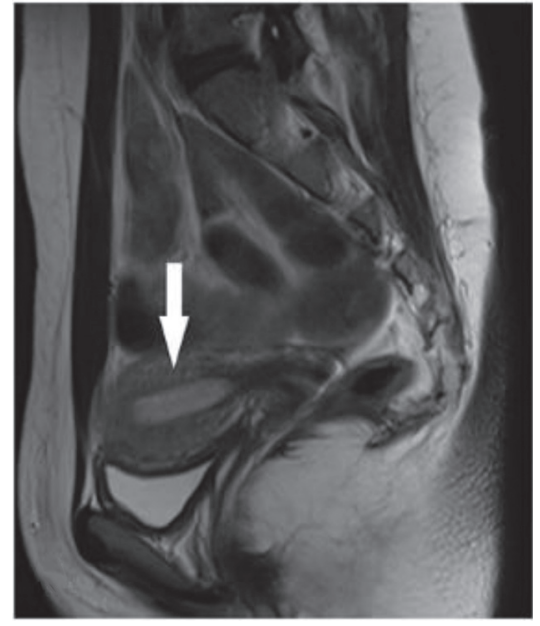

(a)

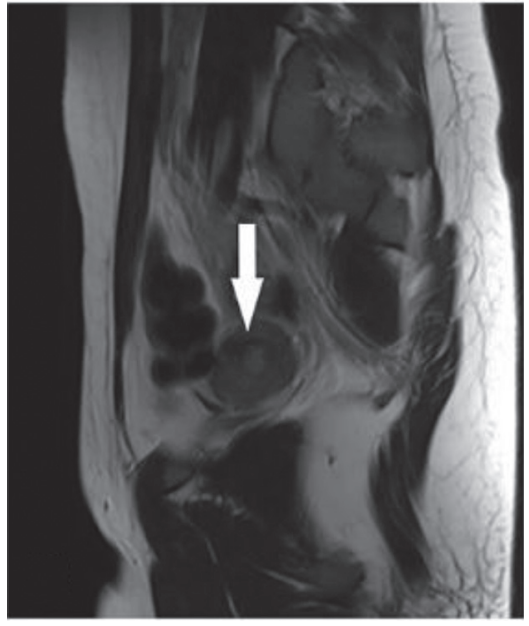

(b)

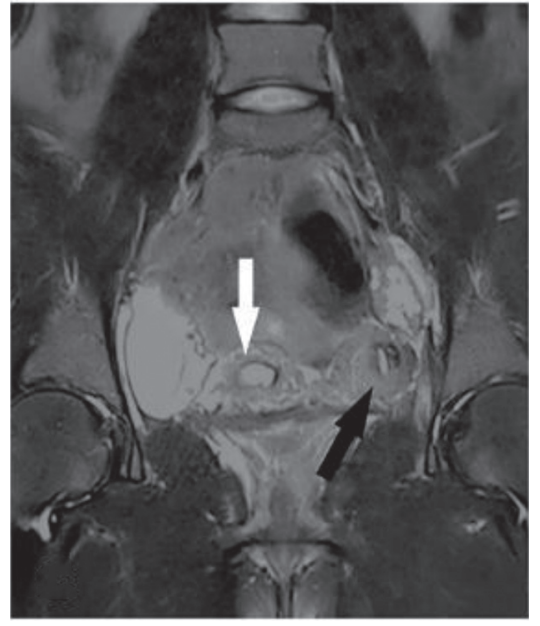

(c)

Figure 4: (a, b) Sagittal T2-weighted images of the pelvis reveal the uterus and the accessory and cavitated uterine mass, respectively. (c) Coronal T2-weighted image of the pelvis demonstrates the uterus on the right side (white arrow) and the accessory and cavitated uterine mass on the left side (black arrow), which are separate from each other.

TABLE 1: Summary of previously reported cases of ACUM regarding their clinical features and management.

\begin{tabular}{|c|c|c|c|c|}
\hline Reference & $\begin{array}{l}\text { Age at } \\
\text { diagnosis } \\
\text { (years) }\end{array}$ & Symptoms & Preliminary diagnosis & Type of operation \\
\hline Present study & (a) 14 & $\begin{array}{l}\text { (a) Chronic pelvic pain for two years } \\
\text { (a) A 3-month history of severe pain }\end{array}$ & $\begin{array}{l}\text { Para-ovarian mass or myoma } \\
\text { (a) Endometrioma or unicornuate }\end{array}$ & Laparotomy \\
\hline $\begin{array}{l}\text { Supermaniam } \\
\text { et al. [15] }\end{array}$ & $\begin{array}{l}\text { (a) } 22 \\
\text { (b) } 36\end{array}$ & $\begin{array}{l}\text { after menses } \\
\text { (b) Chronic pelvic pain and severe } \\
\text { dysmenorrhea since menarche }\end{array}$ & $\begin{array}{l}\text { uterus with a noncommunicating } \\
\text { rudimentary horn } \\
\text { (b) ACUM or degenerating fibroid }\end{array}$ & $(\mathrm{a}, \mathrm{b})$ Laparoscopy \\
\hline Paul et al. [2] & $\begin{array}{l}\text { (a) } 19 \\
\text { (b) } 17 \\
\text { (c) } 25\end{array}$ & $\begin{array}{l}\text { (a) Chronic lower abdominal pain and } \\
\text { severe dysmenorrhea for } 4 \text { years } \\
\text { (b) Dysmenorrhea for } 2 \text { years } \\
\text { (c) Dysmenorrhea for } 7 \text { years }\end{array}$ & $\begin{array}{l}\text { (a) Uterine bicornis with a right horn } \\
\text { hematometra } \\
\text { (b) N/A } \\
\text { (c) An obstructed rudimentary horn }\end{array}$ & $(a, b, c)$ Laparoscopy \\
\hline
\end{tabular}


TABle 1: Continued.

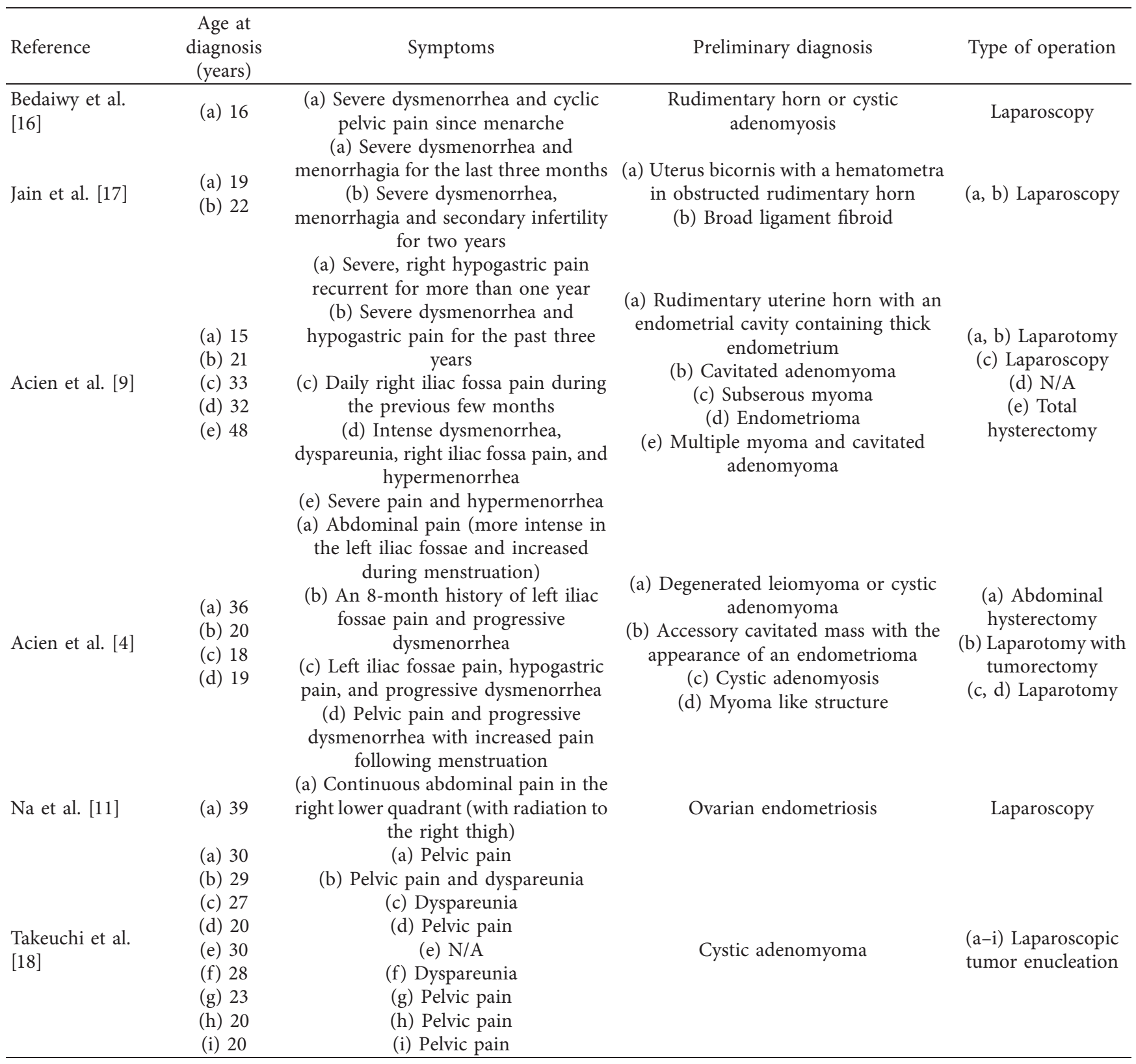

N/A, not available; ACUM, accessory and cavitated uterine mass.

that may occur before, during, or after menstruation [4]. Affected individuals usually experience severe cyclic pelvic pain and dysmenorrhea due to the presence of functional accessory endometrium [10].

Patients usually undergo surgical excision of the mass, and most of them have a presumed diagnosis of adnexal mass or pedunculated myoma preoperatively [11]. However, ACUM is diagnosed when an isolated cavitated mass is resected via surgery in patients with otherwise normal uterus, fallopian tubes, and ovaries [19].

Conclusively, ACUM is a rare Mullerian anomaly and treatable cause of severe dysmenorrhea in young females. To the best of our knowledge, most cases, as ours, are misdiagnosed before surgery, and diagnosis can only be confirmed after histopathological examination; hence, we recommend imaging modalities such as MRI for better evaluation. Pelvic MRI is highly accurate in making a diagnosis of ACUM in young women who present with early-onset dysmenorrhea and recurrent periodic pelvic pain, especially with otherwise normal uterus and ovaries. Although the true prevalence of ACUMs is not known yet, we believe that this clinical entity is in fact more common than currently thought. Thus, ACUM should be considered in the differential diagnosis of patients with clinically relevant symptoms. 


\section{Abbreviations:}

ACUM: Accessory and cavitated uterine mass

MRI: Magnetic resonance imaging

OCP: Oral contraceptive pill

ULM: Uterine-like mass.

\section{Data Availability}

The data and material used in this study will be available from the corresponding author upon request.

\section{Ethical Approval}

All procedures followed the ethical standards of the 1975 Declaration of Helsinki, as revised in 2000. Institutional review board approval was obtained for the conduct of this study.

\section{Consent}

Written informed consent was obtained from the patient for publication of this case report and accompanying images.

\section{Conflicts of Interest}

The authors declare that there is no conflict of interest regarding the publication of this article.

\section{References}

[1] A. Garofalo, M. G. Alemanno, O. Sochirca et al., "Accessory and cavitated uterine mass in an adolescent with severe dysmenorrhoea: from the ultrasound diagnosis to surgical treatment," Journal of Obstetrics and Gynaecology: The Journal of the Institute of Obstetrics and Gynaecology, vol. 37, no. 2, pp. 259-261, 2017.

[2] P. G. Paul, G. Chopade, T. Das, N. Dhivya, S. Patil, and M. Thomas, "Accessory cavitated uterine mass: a rare cause of severe dysmenorrhea in young women," Journal of Minimally Invasive Gynecology, vol. 22, no. 7, pp. 1300-1303, 2015.

[3] O. Koukoura, E. Kapsalaki, A. Daponte, and G. Pistofidis, "Laparoscopic treatment of a large uterine cystic adenomyosis in a young patient," BMJ Case Reports, vol. 22, 2015.

[4] P. Acién, A. Bataller, F. Fernández, M. I. Acién, J. M. Rodríguez, and M. J. Mayol, "New cases of accessory and cavitated uterine masses (ACUM): a significant cause of severe dysmenorrhea and recurrent pelvic pain in young women," Human Reproduction, vol. 27, no. 3, pp. 683-694, 2012.

[5] D. Wilson and B. Bordoni, Embryology, Mullerian Ducts (Paramesonephric Ducts), StatPearls Publishing, Treasure Island, FL, USA, 2020.

[6] M. A. Friedman, L. Aguilar, Q. Heyward, C. Wheeler, and A. Caldamone, "Screening for Mullerian anomalies in patients with unilateral renal agenesis: leveraging early detection to prevent complications," Journal of Pediatric Urology, vol. 14, no. 2, pp. 144-149, 2018.

[7] G. F. Grimbizis, S. Gordts, A. Di Spiezio Sardo et al., "The ESHRE-ESGE consensus on the classification of female genital tract congenital anomalies," Gynecological Surgery, vol. 10, no. 3, pp. 199-212, 2013.
[8] P. Acién, "REVIEW: embryological observations on the female genital tract," Human Reproduction, vol. 7, no. 4, pp. 437-445, 1992.

[9] P. Acién, M. Acién, F. Fernández, M. José Mayol, and I. Aranda, "The cavitated accessory uterine mass," Obstetrics \& Gynecology, vol. 116, no. 5, pp. 1101-1109, 2010.

[10] N. Jain and R. Verma, "Imaging diagnosis of accessory and cavitated uterine mass, a rare mullerian anomaly," Indian Journal of Radiology and Imaging, vol. 24, no. 2, pp. 178-181, 2014.

[11] K. Y. Na, G. Y. Kim, K. Y. Won et al., "Extrapelvic uterus-like masses presenting as colonic submucosal tumor: a case study and review of literature," Korean Journal of Pathology, vol. 47, no. 2, pp. 177-181, 2013.

[12] S. Gordts, R. Campo, and I. Brosens, "Hysteroscopic diagnosis and excision of myometrial cystic adenomyosis," Gynecological Surgery, vol. 11, no. 4, pp. 273-278, 2014.

[13] N. Peyron, E. Jacquemier, M. Charlot et al., "Accessory cavitated uterine mass: MRI features and surgical correlations of a rare but under-recognised entity," European Radiology, vol. 29, no. 3, pp. 1144-1152, 2019.

[14] V. Malhotra, S. Dahiya, S. Nanda, M. Chauhan, and V. Bhuria, "Accessory and cavitated uterine mass: is it a müllerian-duct anomaly?" Journal of Gynecologic Surgery, vol. 2, 2020.

[15] A. Peters, N. B. Rindos, R. S. Guido, and N. M. Donnellan, "Uterine-sparing laparoscopic resection of accessory cavitated uterine masses," Journal of Minimally Invasive Gynecology, vol. 25, no. 1, pp. 24-25, 2018.

[16] S. Supermaniam and W. L. Thye, "Diagnosis and laparoscopic excision of accessory cavitated uterine mass in young women: two case reports," Case Reports in Women's Health, vol. 26, Article ID e00187, 2020.

[17] M. A. Bedaiwy, D. N. Henry, S. Elguero, S. Pickett, and M. Greenfield, "Accessory and cavitated uterine mass with functional endometrium in an adolescent: diagnosis and laparoscopic excision technique," Journal of Pediatric and Adolescent Gynecology, vol. 26, no. 4, pp. e89-e91, 2013.

[18] N. Jain and S. Goel, "Cystic Adenomyoma simulates uterine malformation: a diagnostic dilemma: case report of two unusual cases," Journal of Human Reproductive Sciences, vol. 5, no. 3, pp. 285-288, 2012.

[19] H. Takeuchi, M. Kitade, I. Kikuchi, J. Kumakiri, K. Kuroda, and M. Jinushi, "Diagnosis, laparoscopic management, and histopathologic findings of juvenile cystic adenomyoma: a review of nine cases," Fertility and Sterility, vol. 94, no. 3, pp. 862-868, 2010. 